\title{
Examination of Sufficient Conditions for Forming Mass of "Massive Graviton", from Early Universe
}

\author{
Andrew Walcott Beckwith \\ Physics Department, College of Physics, Chongqing University Huxi Campus, Chongqing, China \\ Email: Rwill9955b@gmail.com, abeckwith@uh.edu
}

How to cite this paper: Beckwith, A.W. (2017) Examination of Sufficient Conditions for Forming Mass of "Massive Graviton", from Early Universe. Journal of High Energy Physics, Gravitation and Cosmology, 3, 34-38.

http://dx.doi.org/10.4236/jhepgc.2017.31006

Received: July 11, 2016

Accepted: November 21, 2016

Published: November 25, 2016

Copyright ( $) 2017$ by author and Scientific Research Publishing Inc. This work is licensed under the Creative Commons Attribution International License (CC BY 4.0).

http://creativecommons.org/licenses/by/4.0/

\begin{abstract}
We start with a formulation of a modified "Poisson" equation from Poissons and Will as of 2014, and then use the Padmanabhan inter relationship between an inflaton and an early universe potential system. Then from there, we come up with a quadratic equation for a minimum radius, for producing a "massive graviton" value. We then close with observations as to what this implies as to gravitational physics.
\end{abstract}

\section{Keywords}

Modified Poisson Equation, Massive Gravity, Inflaton Physics

\section{What Is Important about the Modified Poissons Equation [1]?}

We will first of all refer to two necessary and sufficient conditions for the onset of a massive graviton given in [1], and combined with Padmanablan's reference [2].

i.e. what we will be doing is to re do the reference calculations given in [1] with

$$
\left(\nabla^{2}+\left[\lambda^{-2}=\left(\frac{m_{\text {graviton }} c}{\hbar}\right)^{2}\right]\right)\left[U=\frac{G m}{r} \cdot \exp \left[(-r / \lambda)=\left(\frac{r \cdot m_{\text {graviton }} c}{\hbar}\right)\right]\right]=-4 \pi G \rho
$$

Here, we will be using in the Pre Planckian potential the inputs from the data usually associated with [2]

$$
\begin{aligned}
& a \approx a_{\min } t^{\gamma} \\
& \Leftrightarrow \phi \approx \sqrt{\frac{\gamma}{4 \pi G}} \cdot \ln \left\{\sqrt{\frac{8 \pi G V_{0}}{\gamma \cdot(3 \gamma-1)}} \cdot t\right\} \\
& \Leftrightarrow V \approx V_{0} \cdot \exp \left\{-\sqrt{\frac{16 \pi G}{\gamma}} \cdot \phi(t)\right\}
\end{aligned}
$$

In other words, we will be using the inflation given by 


$$
\phi \approx \sqrt{\frac{\gamma}{4 \pi G}} \cdot \ln \left\{\sqrt{\frac{8 \pi G V_{0}}{\gamma \cdot(3 \gamma-1)}} \cdot t\right\}
$$

If so, then our approximation is to call the Potential in Equation (2) to be the same as $U$ in Equation (1), and then with re arrangements we come up with the following

$$
\left[\frac{\mathrm{d}^{2}}{\mathrm{~d} r^{2}}+\left(\frac{m_{\text {graviton }} c}{\hbar}\right)^{2}\right] \cdot\left(\frac{r^{-1} \alpha \cdot(3 \alpha-1)}{32 \pi^{2}}\right)=G \cdot \rho
$$

Then, after algebra, we have the following

$$
\left(m_{\text {graviton }}\right)^{2} \approx\left[\frac{32 \pi^{2} r \cdot \hbar^{2} \cdot G \cdot \rho}{c^{2} \cdot \alpha \cdot(3 \alpha-1)}-\frac{16 \pi^{2} r^{-1} \cdot \hbar^{2} \cdot G^{-2}}{c^{2} \cdot \alpha \cdot(3 \alpha-1)}\right]
$$

The quadratic Equation this engenders is, how to say

$$
\begin{aligned}
& \left(m_{\text {graviton }}\right)^{2} \approx\left[\frac{32 \pi^{2} r \cdot \hbar^{2} \cdot G \cdot \rho}{c^{2} \cdot \alpha \cdot(3 \alpha-1)}-\frac{16 \pi^{2} r^{-1} \cdot \hbar^{2} \cdot G}{c^{2} \cdot \alpha \cdot(3 \alpha-1)}\right] \\
& \Rightarrow r^{2}-\frac{r \cdot\left(m_{\text {graviton }}\right)^{2}}{\left(\frac{32 \pi^{2} \hbar^{2} \cdot G \cdot \rho}{c^{2} \cdot \alpha \cdot(3 \alpha-1)}\right)}-\frac{\left(\frac{16 \pi^{2} \cdot \hbar^{2} \cdot G}{c^{2} \cdot \alpha \cdot(3 \alpha-1)}\right)}{\left(\frac{32 \pi^{2} \hbar^{2} \cdot G \cdot \rho}{c^{2} \cdot \alpha \cdot(3 \alpha-1)}\right)} \\
& \quad \doteq r^{2}-\frac{r \cdot\left(m_{\text {graviton }}\right)^{2}}{\left(\frac{32 \pi^{2} \hbar^{2} \cdot G \cdot \rho}{c^{2} \cdot \alpha \cdot(3 \alpha-1)}\right)}-\frac{1}{2 \rho}=0
\end{aligned}
$$

A candidate for the density functional will come next, with the way of obtaining a critical value for $r$.

\section{Density Functional Inserted into Equation (6)}

In [3] we make the assumption, namely

$$
V_{\text {Pre-Planckian }} \sim\left(\Delta E \sim \frac{\hbar}{\delta t \cdot a_{\text {min }}^{2} \phi_{\text {inf }}}\right)
$$

As far as applications to: [1]

$$
\begin{aligned}
& r^{2}-\frac{r^{4} \cdot\left(m_{\text {graviton }}\right)^{2} a_{\text {min }}^{2} \cdot \delta t \cdot \sqrt{\frac{\gamma}{4 \pi G} \cdot \ln \left\{\sqrt{\frac{8 \pi G V_{0}}{\gamma \cdot(3 \gamma-1)}} \cdot t\right\}}}{\left(\frac{32 \pi^{2} \hbar^{2} \cdot G}{c^{2} \cdot \alpha \cdot(3 \alpha-1)}\right)} \\
& -\frac{r^{3} a_{\text {min }}^{2} \delta t \cdot \sqrt{\frac{\gamma}{4 \pi G}} \cdot \ln \left\{\sqrt{\frac{8 \pi G V_{0}}{\gamma \cdot(3 \gamma-1)}} \cdot t\right\}}{2}=0
\end{aligned}
$$

Then if we use $\phi \approx \sqrt{\frac{\gamma}{4 \pi G}} \cdot \ln \left\{\sqrt{\frac{8 \pi G V_{0}}{\gamma \cdot(3 \gamma-1)}} \cdot t\right\}$ 


$$
\begin{aligned}
& r^{2}-\frac{r^{4} \cdot\left(m_{\text {graviton }}\right)^{2} a_{\min }^{2} \cdot \delta t \cdot \phi}{\left(\frac{32 \pi^{2} \hbar^{2} \cdot G}{c^{2} \cdot \alpha \cdot(3 \alpha-1)}\right)}-\frac{r^{3} a_{\min }^{2} \delta t \cdot \phi}{2}=0 \\
& \Rightarrow 1-\frac{r^{2} \cdot\left(m_{\text {graviton }}\right)^{2} a_{\min }^{2} \cdot \delta t \cdot \phi}{\left(\frac{32 \pi^{2} \hbar^{2} \cdot G}{c^{2} \cdot \alpha \cdot(3 \alpha-1)}\right)}-\frac{r \cdot a_{\min }^{2} \delta t \cdot \phi}{2}=0
\end{aligned}
$$

Then if we are looking at extremely small times in the inflaton, the above becomes

$$
1+\frac{r^{2} \cdot\left(m_{\text {graviton }}\right)^{2} a_{\min }^{2} \cdot \delta t \cdot|\phi|}{\left(\frac{32 \pi^{2} \hbar^{2} \cdot G}{c^{2} \cdot \alpha \cdot(3 \alpha-1)}\right)}+\frac{r \cdot a_{\min }^{2} \delta t \cdot|\phi|}{2}=0
$$

Or

$$
r^{2}+\frac{\left(\frac{32 \pi^{2} \hbar^{2} \cdot G}{c^{2} \cdot \alpha \cdot(3 \alpha-1)}\right)}{\left(m_{\text {graviton }}\right)^{2}} \cdot r+\frac{\left(\frac{32 \pi^{2} \hbar^{2} \cdot G}{c^{2} \cdot \alpha \cdot(3 \alpha-1)}\right)}{\left(m_{\text {graviton }}\right)^{2} a_{\text {min }}^{2} \cdot \delta t \cdot|\phi|}=0
$$

We claim, that warts and all, this is a first order approximation as to the distance, from the moment of creation, for definitive acquisition for a "mass" to a massive graviton, and it is a definitive restraint.

\section{Conclusion. A Very Strange, Not Necessarily Real Valued Initial Radial Condition}

Possibly to incorporate some sort of measurement protocol, for Equation (8) this equation would have to be the absolute magnitude. This has yet to be determined in discussions the author will have with members of the HFGW in Chongqing University. But what is noticeable, is that the inflaton equation as given by Padmanabhan [2] hopefully will not be incommensurate with the physics of the Corda Criteria given in the Gravity's breath document [4]. Keep in mind the importance of the result from reference [5] below which forms the core of Equation (12) below

$$
N_{e \text {-foldings }}=-\frac{8 \pi}{m_{\text {Planck }}^{2}} \cdot \int_{\phi_{1}}^{\phi_{2}} \frac{V(\phi)}{\left(\frac{\partial V(\phi)}{\partial \phi}\right)} \mathrm{d} \phi \geq 65
$$

We should also attempt to assure fidelity with Equation (8) above, in what work we are doing.

Furthermore, we should keep in mind the physics incorporated in [6] [7], i.e. as to the work of LIGO. i.e. it is important to keep in mind that in addition, that [8] has confirmed that a subsequent analysis of the event GW150914 by the LSC constrained the graviton Compton wavelength of those alternative theories of gravity in which the graviton is massive and placed a level of $90 \%$ confidence on the lower bound of $10^{\wedge}\{13\}$ $\mathrm{km}$ for a Compton wavelength of the graviton. This will in its own way subsequently add additional rigor to the analysis of the graviton mass stated in Equation (9) as well as the Compton wavelength lower bound as given in Equation (1) above. 
Doing these sort of vetting protocols is the same as with being consistent with investigation as to a real investigation as to the fundamental nature of gravity. i.e. is this a way to show if general relativity is the final theory of gravitation. i.e., if massive gravity is confirmed, as given in [9], then GR is perhaps to be replaced by a scalar-tensor theory, as has been shown by Corda.

In addition, note that [10] references how, in terms of refinement of gravitational wave detectors, that second generation gravitational wave detectors require high power lasers with several 100W of output power and with very low temporal and spatial fluctuations would be helpful in terms of resolution perhaps aiding in obtaining resolution of the graviton mass as given in [8], which is in turn also commensurate with [11], as far as precise resolution of massive gravity's foot print, which in turn will aid in using Corda's insights as given in [9].

\section{Acknowledgements}

This work is supported in part by National Nature Science Foundation of China grant No. 11375279.

\section{References}

[1] Poisson, E. and Will, C. (2014) Gravity, Newtonian, Post Newtoninan, Relativistic. Cambridge University Press, Cambridge, UK. https:/doi.org/10.1017/CBO9781139507486

[2] Padmanabhan, T. (2005) Understanding Our Universe, Current Status and Open Issues. In: Ashatekar, A., Ed., 100 Years of Relativity, Space-Time Structure: Einstein and Beyond, World Scientific Publishing Co. Pte. Ltd., Singapore, 175-204. http://arxiv.org/abs/gr-qc/0503107

[3] Beckwith, A. (2016) Gedanken Experiment for Refining the Unruh Metric Tensor Uncertainty Principle via Schwarzschild Geometry and Planckian Space-Time with Initial Nonzero Entropy and Applying the Riemannian-Penrose Inequality and Initial Kinetic Energy for a Lower Bound to Graviton Mass (Massive Gravity). Journal of High Energy Physics, Gravitation and Cosmology, 2, 106-124. https:/doi.org/10.4236/jhepgc.2016.21012

[4] Corda, C. (2012) Gravity's Primordial Breath. Electronic Journal of Theoretical Physics, EJTP, 9, 1-10. http://www.ejtp.com/articles/ejtpv9i26p1.pdf

[5] Freese, K. (1992) Natural Inflation. In: Nath, P. and Recucroft, S., Eds., Particles, Strings, and Cosmology, Northeastern University, 25-30 March 1991, World Scientific Publishing company, Pte. Ltd., Singapore, 408-428.

[6] Abbott, B. P., et al. (LIGO Scientific Collaboration and Virgo Collaboration) (2016) Observation of Gravitational Waves from a Binary Black Hole Merger. Physical Review Letters, 116, Article ID: 061102.

[7] Abbott, B.P., et al., LIGO Scientific Collaboration and Virgo Collaboration (2016) GW151226: Observation of Gravitational Waves from a 22-Solar-Mass Binary Black Hole Coalescence. Physical Review Letters, 116, Article ID: 241103.

[8] Abbott, B.P., et al. Tests of general relativity with GW150914. https://arxiv.org/pdf/1602.03841.pdf

[9] Corda, C. (2009) Interferometric Detection of Gravitational Waves: The Definitive Test for General Relativity. International Journal of Modern Physics D, 18, 2275-2282. https://arxiv.org/abs/0905.2502 https:/doi.org/10.1142/S0218271809015904

[10] Willke, B, et al. Stabilized High Power Laser for Advanced Gravitational Wave Detectors. 
http://iopscience.iop.org/article/10.1088/1742-6596/32/1/040/meta;jsessionid=B072B9D72E 7EB4F993D70061EEE365CF.c5.iopscience.cld.iop.org

[11] Flaminico, R. et al. (2010) A Study of Coating Mechanical and Optical Losses in View of Reducing Mirror Thermal Noise in Gravitational Wave Detectors. Classical and Quantum Gravity, 27, Article ID: 084030. https:/doi.org/10.1088/0264-9381/27/8/084030

Submit or recommend next manuscript to SCIRP and we will provide best service for you:

Accepting pre-submission inquiries through Email, Facebook, LinkedIn, Twitter, etc. A wide selection of journals (inclusive of 9 subjects, more than 200 journals) Providing 24-hour high-quality service User-friendly online submission system Fair and swift peer-review system Efficient typesetting and proofreading procedure Display of the result of downloads and visits, as well as the number of cited articles Maximum dissemination of your research work

Submit your manuscript at: http://papersubmission.scirp.org/

Or contact jhepgc@scirp.org 